\title{
Microstructure of Bone Around Natural Hole in Bovine Lumbar Vertebra"
}

\author{
Bijay GIRI ${ }^{* *}$, Shigeru TADANO ${ }^{* *}$, Kazuhiro FUJISAKI**, Masahiro TODOH ${ }^{* *}$ \\ ${ }^{* *}$ Division of Human Mechanical Systems and Design, \\ Graduate School of Engineering, \\ Hokkaido University \\ N13 W8, Kita-ku, Sapporo 060-8628, Japan \\ E-mail: bijay@mech-me.eng.hokudai.ac.jp
}

\begin{abstract}
Naturally occurring holes in bone called foramina are not detected as region of crack initiation or growth. Lumbar vertebrae, which are subjected to complex external loads, have considerable number of such big and small foramina. The mechanical characteristics of bone profoundly depend on the mineral content (HAp crystals) and collagen fibers alignment, where c-crystallographic axis of HAp crystals are preferentially oriented parallel to the long axis of collagen fibers. In the current work, we investigated the crystal orientation and mineral distribution around the foramen using X-rays to explore the reason of foramen not being region of crack initiation or growth to the precise level. Specimens were harvested from bovine lumbar vertebrae. The orientations of HAp crystals were determined from the diffracted intensity profile of X-rays using imaging plate. Mineral fractions were measured from the fraction of X-rays transmitted through the specimen. The tangential orientation of the crystals revealed around the foramen edge was considered as the main reinforcing parameter for the foramen. The minerals were distributed in the specimen without any predictable tendency. Hence, they were not considered as a strengthening parameter. A thicker cortical region was discovered near the surface around the upper edge of the foramen, which was also extended within the cancellous mass as a thin cylindrical cortical shell covering the foramen from inside. Such structures were regarded to result the foramen a damage resistant site.
\end{abstract}

Key words: Stress Concentration, Foramen, Bovine Lumbar Vertebra, Cortical Bone, Hydroxyapatite Crystals, X-Ray Diffraction

\section{Introduction}

Discontinuities like holes, slots, grooves, notches, etc introduced in the structures often result in stress concentration and thereby weaken the strength of the structures. Structures with such discontinuities are essential in most engineering designs. Bone also contains holes of varying size in every part of the body. Such holes known as 'Foramina' (singular 'Foramen'), existing for blood vessels and nerves to pass, are generally not found as a region of crack initiation or growth. Vertebral body also contains large-small basivertebral foramina through which internal and external plexuses communicate with each other. We observed considerable number of such big and small foramina in bovine lumbar vertebra. These foramina of lumbar region, which are also subjected to external complex loads, are rarely considered in previous related studies.

Bone, a complex tissue, has hierarchy of structure. At microstructural level, it is composed mainly of two constituents - inorganic mineral part of hydroxyapatite (HAp)

${ }^{*}$ Received 13 June, 2006 (No. 06-0045) [DOI: 10.1299/jbse.2.1] 
crystals and organic part of collageneous matrix. The mineral phase of bone tissue, known as biological apatite, is a pure hydroxyapatite of chemical composition as $\mathrm{Ca}_{10}\left(\mathrm{PO}_{4}\right)_{6}(\mathrm{OH})_{2}$ with some impurities and having hexagonal crystallographic structures. The widely accepted basic structure of bone tissue is a collagen-crystal composite, where the HAp crystals are embedded in the gaps inside the collagen matrix. These crystals grow with specific crystalline orientation. The preferred orientation of c-crystallographic axis of the crystals, i.e. the axis of symmetry parallel to the longer dimension of crystal, also called as 002-lattice plane, is parallel to the long axis of collagen fibers ${ }^{(1)}{ }^{(2)}$. Furthermore, the mechanical properties of bone depend on its compositional parameters - mainly mineral content and porosity, and structure i.e. organization of collagens ${ }^{(3)}$.

In the current investigation, we applied latest X-ray methods to precisely reveal microstructure of bone around the foramen of bovine lumbar vertebra existing in the scale of millimeters. We attempted to explore the reason of stiffer foramen considering the orientation of crystals and distribution of minerals around the foramen. The diffracted intensity profile of X-rays was used to measure the orientation of crystals. The fraction of transmitted intensity was quantified to determine the distribution of mineral (crystals) fraction. We also explored the internal structure of foramen macroscopically as well as microscopically. Our curiosity in the vertebral bone was to know how bone adapts with the foramen in this part in spite of thin cortical region and additionally facing multiple types of loads compared to other parts.

\section{Materials and Methods}

\subsection{Specimen}

Three spine samples, all from 24 months old (female) bovine donors, were obtained. The vertebrae were separated from the spines and frozen at $-30^{\circ} \mathrm{C}$ until further preparation.

Two L6 vertebrae and one L5 vertebra were selected to harvest cortical specimens from the ventral side with the foramen as shown in Fig. 1; all from different bovine samples. Since the cortical part in the vertebra body is very thin, it was difficult to obtain the cortical slice at a single attempt. Flat rectangular sections of the cortical bone with the foramen almost lying at the centre were obtained by series of cutting, grinding and polishing. The steps we applied to obtain the final specimens have been illustrated in Fig. 2. All the rough cuts were done using hand-saw and finished cuts using circular diamond blade saw. The surfaces of the specimens were gradually smoothed in series of emery papers and polished in diamond paste. The samples were washed in the ultrasonic bath with physiological saline during every step of grinding and polishing. Precaution was taken to avoid heating of the specimens during all the steps.

These cortical specimens were used to measure the crystal orientation and mineral distribution. Shape of the foramina in all the three specimens were almost elliptical with major axis aligned along the vertebral axis. Size of the specimens and foramina are given in Table 1. Rectangular regions of the specimen keeping foramen almost at the centre (Fig. 1) were experimented with X-rays from the final prepared specimen. In the case of specimen $\mathrm{B}$, since the vertebral body is curved at the position of foramen, it was divided into two parts (upper and lower) to obtain flat sections.

One more L5 vertebra was used to obtain sections through the foramen for the study of internal structure of the hole. Sections through the centre and end of the foramen were prepared for the examination. 


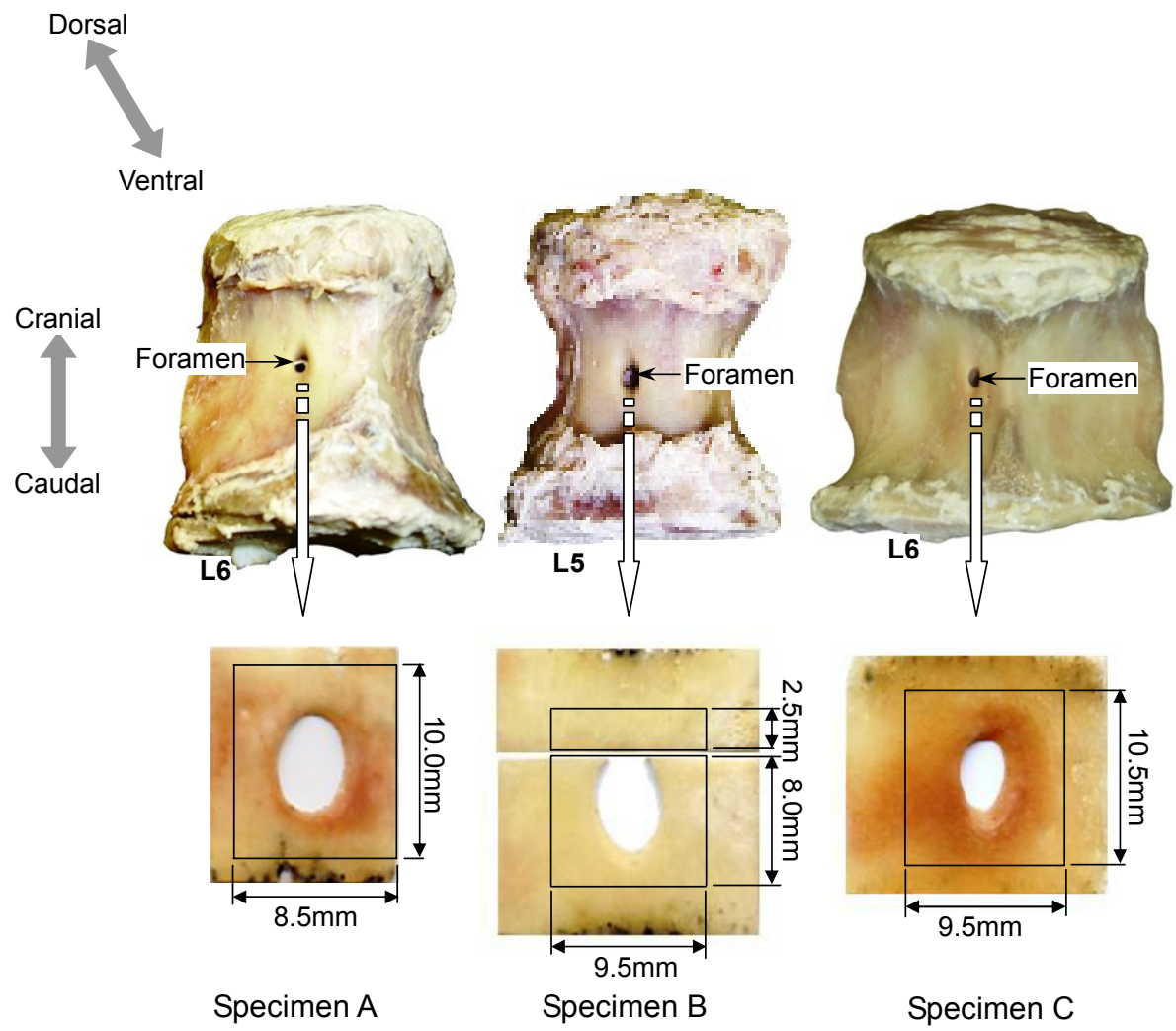

Fig. 1 Specimens containing foramen (bottom) harvested from the vertebrae samples (top). Rectangles in the specimens represent the experimented areas.

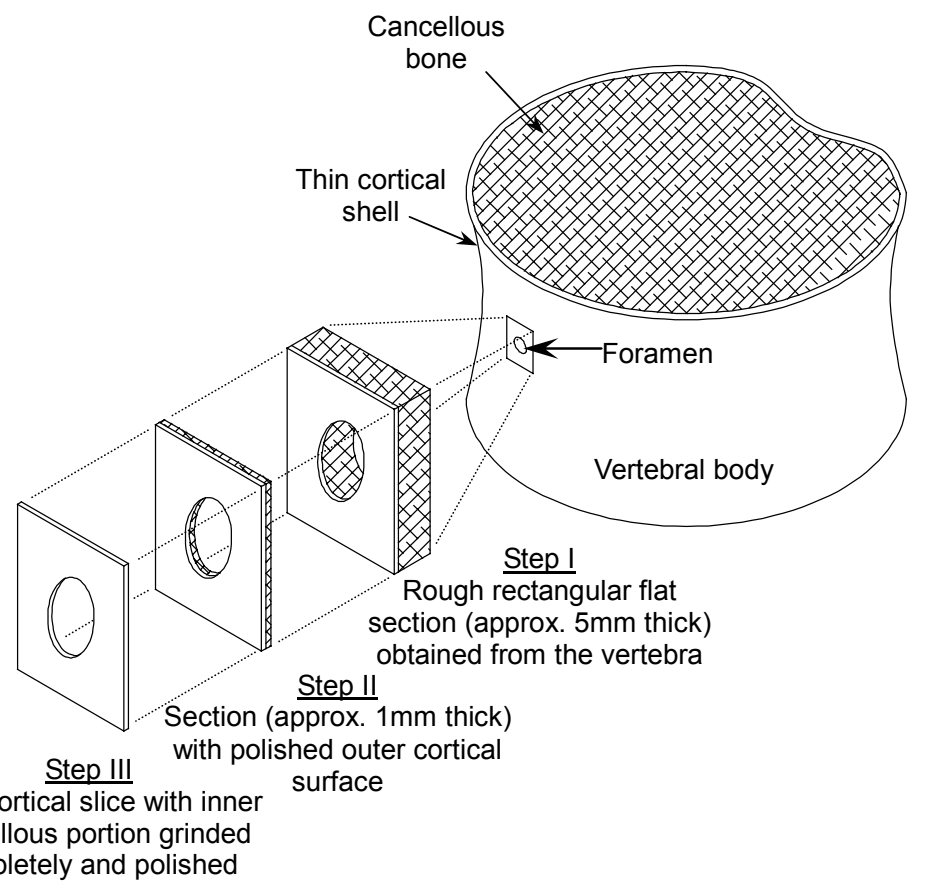

Fig. 2 Final cortical specimen (slice) obtained by series of cutting, grinding and polishing (figure not to scale) 
Table 1 Size of Specimens and Foramina

\begin{tabular}{c|c|r|c|c|c|c|c}
\hline \multirow{2}{*}{ Specimens } & \multirow{2}{*}{$\begin{array}{c}\text { Thickness } \\
(\mathrm{mm})\end{array}$} & \multicolumn{2}{|c|}{ Specimen Size } & \multicolumn{2}{|c|}{$\begin{array}{c}\text { Experimented } \\
\text { area }\end{array}$} & \multicolumn{2}{|c}{$\begin{array}{c}\text { Foramen size } \\
(\mathrm{mm})\end{array}$} \\
\cline { 3 - 8 } & $\begin{array}{c}\text { Height } \\
(\mathrm{mm})\end{array}$ & $\begin{array}{c}\text { Width } \\
(\mathrm{mm})\end{array}$ & $\begin{array}{c}\text { Height } \\
(\mathrm{mm})\end{array}$ & $\begin{array}{c}\text { Width } \\
(\mathrm{mm})\end{array}$ & $\begin{array}{c}\text { Major } \\
\text { axis }\end{array}$ & $\begin{array}{c}\text { Minor } \\
\text { axis }\end{array}$ \\
\hline A & 0.43 & 12.0 & 9.5 & 10.0 & 8.5 & 5.5 & 4.0 \\
B top & 0.62 & 6.0 & 16.0 & 2.5 & 9.5 & 6.0 & 4.0 \\
bottom & 0.66 & 11.0 & 16.0 & 8.0 & 9.5 & 6.0 & 3.5 \\
C & 0.83 & 15.0 & 17.0 & 10.5 & 9.5 & 5.0 & 3.5 \\
\hline
\end{tabular}

\subsection{Determination of Crystal Orientation by $X$-ray Diffraction}

X-rays with characteristics Mo-K $\alpha$, Mo target, wave length $0.071 \mathrm{~nm}$, tube voltage 40 $\mathrm{kV}$ and tube current $40 \mathrm{~mA}$ were generated from RINT2000 X-ray diffractometer (Rigaku Co., Japan). The generated beam was focused perpendicular to the specimen using $0.5 \mathrm{~mm}$ diameter collimator. Rectangular regions of the specimens keeping foramen almost at the center shown in Fig. 1 (also given in Table 1) were experimented. The diffracted radiation through the specimen was absorbed by the imaging plate (BAS-SR $127 \mathrm{~mm}$ by $127 \mathrm{~mm}$, Fuji Photo Film Co. Ltd., Japan). By scanning the imaging plate (using R-axis DS3C scanner, Rigaku Co., Japan), a two dimensional X-ray diffraction pattern was obtained within $1140 \times 1140$ pixels image for one position (i.e. $0.5 \mathrm{~mm}$ diameter area) of the specimen as shown in Fig. 3 (b). Similar intensity distribution patterns were obtained for other positions of the specimen keeping it always vertically parallel to the imaging plate.

A tentative estimate of preferred orientation of the crystals could be made from the dense arc shaped intensity region in 002-lattice plane of the distribution profile (Fig. 3 (b)) (4). To have the exact angle of orientation, we calculated the integrated values of intensity in terms of angles from the intensity distribution in 002-plane strip only using a software program developed in Microsoft Visual Basic. The orientation of crystals was then determined from the maximum value of intensity.

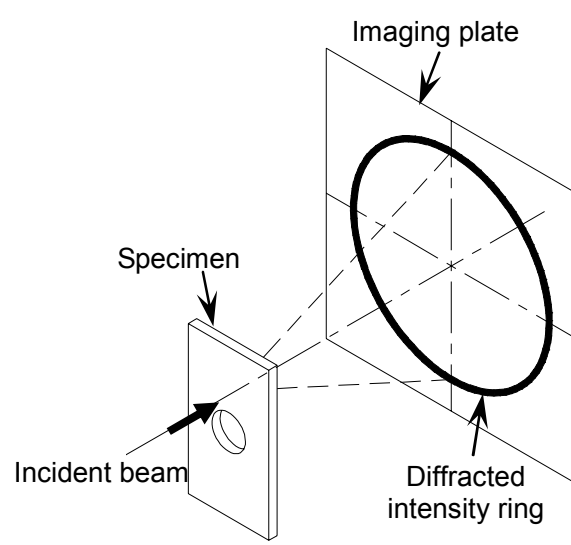

(a)

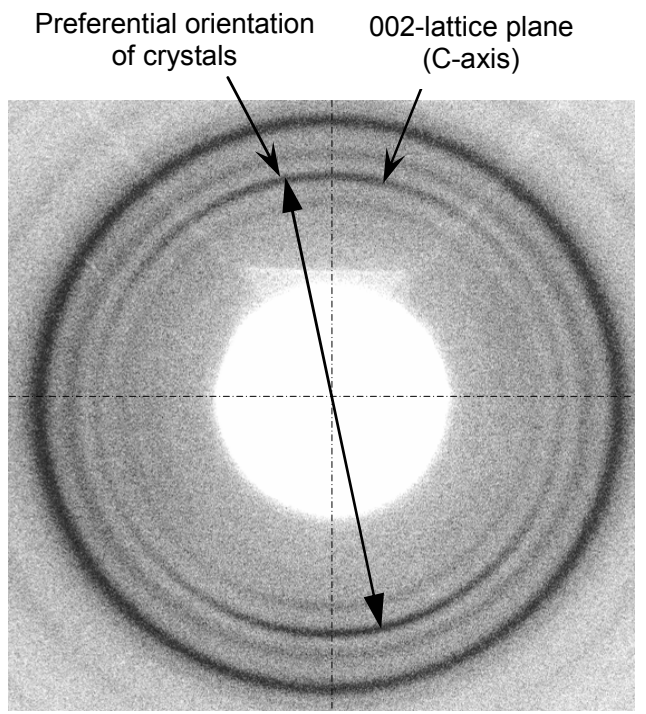

(b)

Fig. 3 (a) Schematic layout of experiment. Specimen and Imaging plate were kept vertically parallel. (b) Diffracted intensity pattern obtained by scanning the imaging plate. Tentative estimate of the orientation could be done by observing the dense arc shaped distribution in 002-lattice plane (c-crystallographic axis). We determined the exact orientation angle by calculating the maximum value of integrated intensity of 002 -plane. 


\subsection{Measurement of Mineral by X-ray Absorption}

Bone is a composite material containing inorganic mineral phase of hydroxyapatite and organic phase of hydrated protein. The inorganic constituent regarded as a crystalline phase (minerals) has intensity absorptance very high compared to the organic matrix. The compositional proportion of such crystalline phase in a multiple phase system can then be determined by X-ray absorption procedure ${ }^{(5)(6)}$.

Characteristic X-rays Ag-Ka, Ag target, wave length $0.056 \mathrm{~nm}$, tube voltage $26 \mathrm{kV}$ and tube current $26 \mathrm{~mA}$ were generated and focused to the specimen using $0.5 \mathrm{~mm}$ diameter collimator. Output pulse of the transmitted intensity was quantified by the scintillation counter. The intensity of transmitted beam $I_{x}$ after passing through the specimen of thickness $x$ is given as ${ }^{(7)}$,

$$
I_{x}=I_{0} \exp \left(-\mu_{b} x\right)
$$

where $I_{0}$ is the intensity of total incident beam and $\mu_{b}$ is the linear absorption coefficient of bone overall.

For bone composite of HAp crystals and collagen, the linear absorption coefficient of bone, $\mu_{b}$ is derived as,

$$
\mu_{b}=v_{h} \mu_{h}+v_{c} \mu_{c}
$$

where $\mu_{h}$ and $\mu_{c}$ are linear absorption coefficients of HAp crystals and collageneous matrix respectively; $v_{h}$ and $v_{c}$ are volume fractions of HAp crystals and collageneous matrix respectively. The value of $\mu_{c}$ being very small compared to $\mu_{h}$ is neglected for the calculation. Calculating $\mu_{b}$ from the average percent transmittance of the intensity using Eq. (1) and putting the value of $\mu_{h}$ equal to $15.0 \mathrm{~cm}^{-1(6)}$, the mineral fraction of HAp crystal $v_{h}$ was calculated using Eq. (2). Since porosity is included in the measurement, the values obtained here represent 'apparent mineral fraction', which has been termed as 'mineral fraction' throughout this article.

\section{Results}

\subsection{Crystal Orientation and Mineral Fraction}

The orientations of crystals around the foramen of the three specimens are shown in Fig. 4. The preferred orientations of the crystals determined from the maximum value of intensity in 002-plane were almost tangential near the edge of the foramen. Irrespective of the vertebral body shape the crystals make a protected layer around the edge, whereas away from the edge they were found to orient irregularly. The crystals were mostly diverted

Specimen A

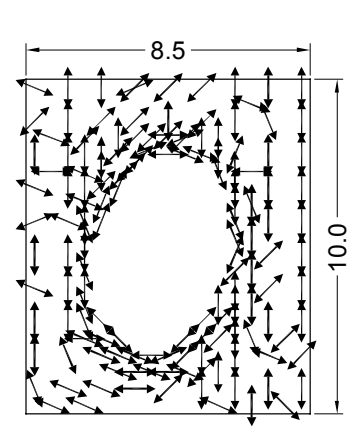

Specimen B

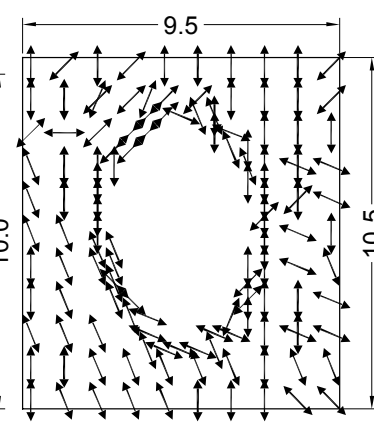

Specimen C

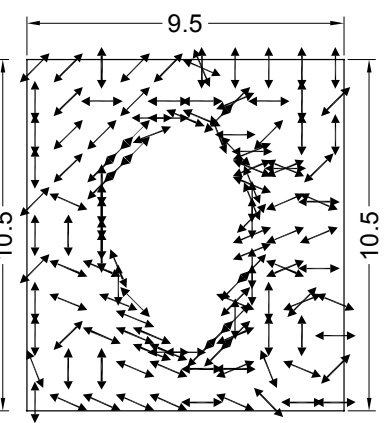

Fig. 4 Preferential orientation of c-axes of crystals determined from the diffracted intensity values. The crystals are mostly tangential around the foramen edge. 

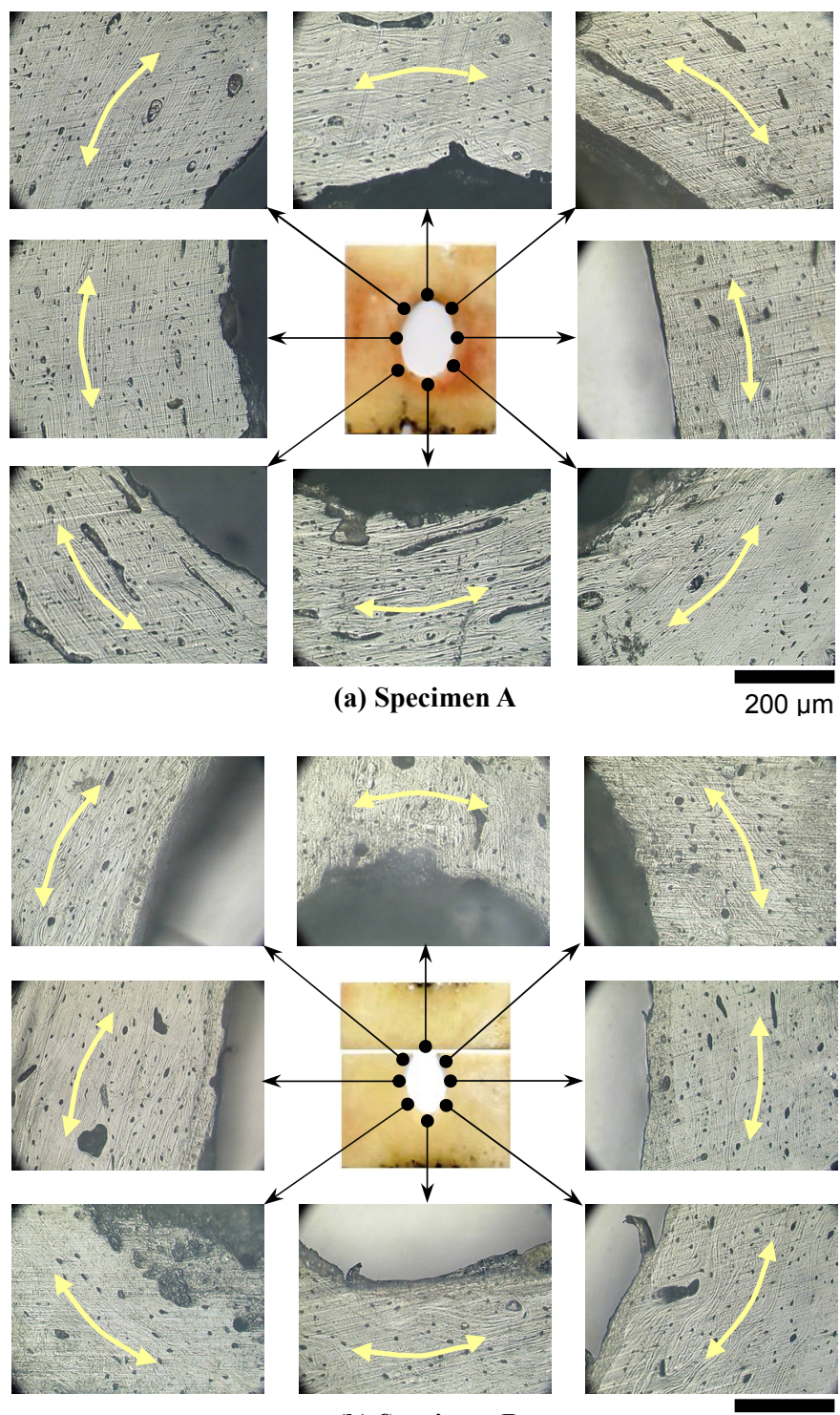

(b) Specimen B

$200 \mu \mathrm{m}$
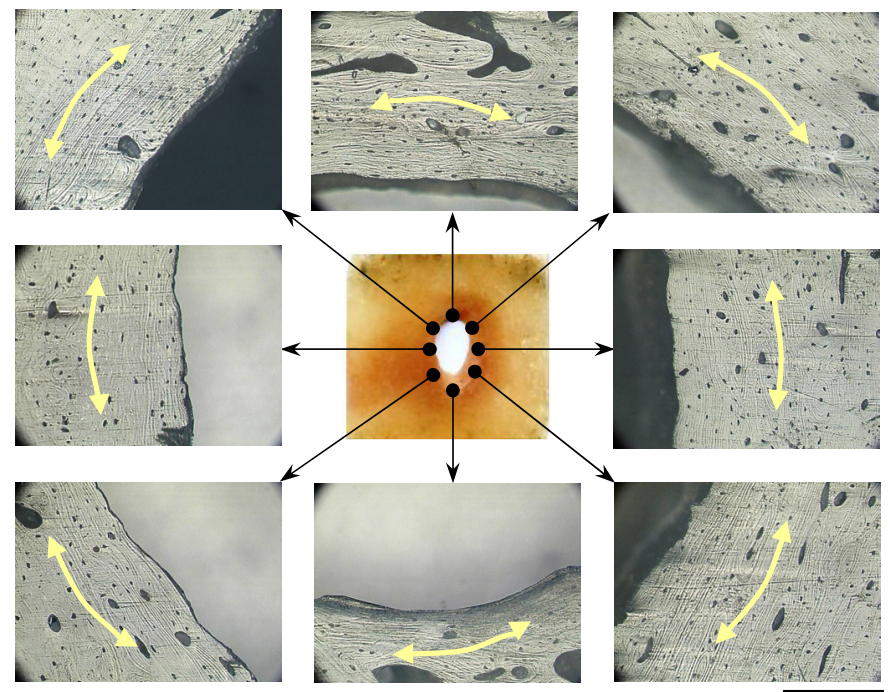

(c) Specimen C
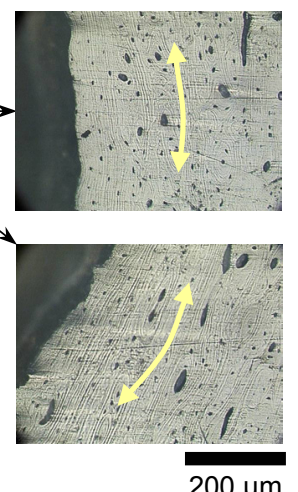

Fig. 5 Microscopic images of (a) specimen A, (b) specimen B, (c) specimen C near the edge of the foramen. Osteons are also tangentially aligned around the foramen. 
depending on the shape of the vertebral body in the location away from the edge instead of being parallel to the longitudinal vertebral axis (Fig. 4). Considering the intensity values within the range of $10 \%$ of the maximum value, more than one possible preferential orientation of the crystals were obtained, represented by multiple arrows at some positions in the figure.

The microscopic images of the locations around the foramen edge also revealed the tangential alignment of osteons (Figs. 5 (a), (b) and (c)). The osteons are almost vertical at right and left part of the foramen, whereas almost horizontal at top and bottom. Similarly, they are diverted with the edge of the foramen keeping tangential at other locations as shown in the figures.

The distributions of mineral fractions are shown in Fig. 6. The mineral fraction values in the specimens were found to be ranging approximately from $20 \%$ to $40 \%$. They were distributed in the specimens with very slight difference in the values having no predictable tendency. No typicality of the distribution in the specimens was found. The lower values (less than 20\%) of the mineral fraction signify the existence of voids in the specimens.

Since we ignored the places with inclined surfaces at the edge (Fig. 1), resulted because of oblique orientation of foramen, the difference in the shape of hole could be noticed in the mineral fraction distribution (Fig. 6). Also, non-uniformity in the shape could be observed as the results represent those from $0.5 \mathrm{~mm}$ intervals.

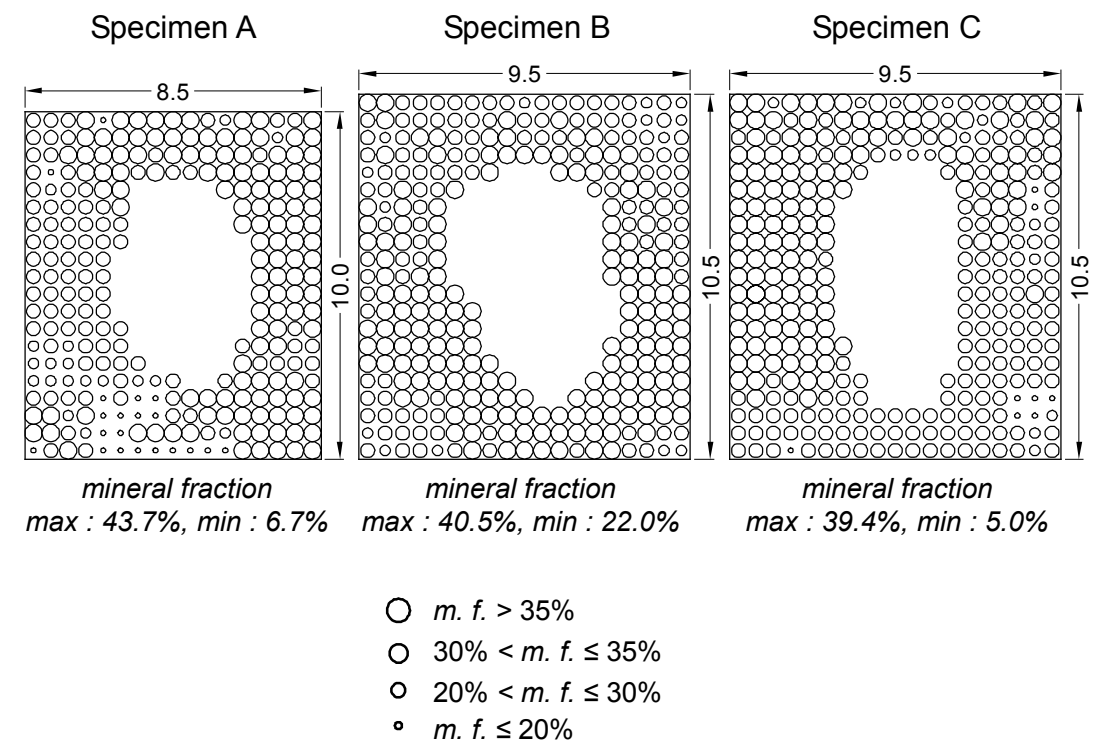

Fig. 6 Distribution of mineral fraction. Diameter of the circle is equivalent to the mineral fraction value. Locations with mineral fraction values less than $20 \%$ are regarded as voids present in the specimens. No specific distribution pattern and no typicality in the specimens could be observed.

\subsection{Bone Section Through the Foramen}

Cortical bone covering the vertebral body existed in a thicker mass near the upper part of the section (external surface of the vertebra) around the foramen edge compared to other regions (Fig. 7 (b)). Further, the same cortical portion was found to continue inside the cancellous mass covering the hole from inside in the form of a thin cylindrical shell (wall) (Fig. 7 (b) and (c)). We also examined the section of bone through the end of foramen containing portion of cortical bone from the cylindrical wall (Fig. 8). The crystals in this portion were found to be horizontally oriented. The clear osteons pack from the mid-section (Fig. 7 (d)) and horizontal orientation from the end-section (Fig. 8 (b)) indicate circumferentially packed crystals in the cylindrical wall around the foramen. 


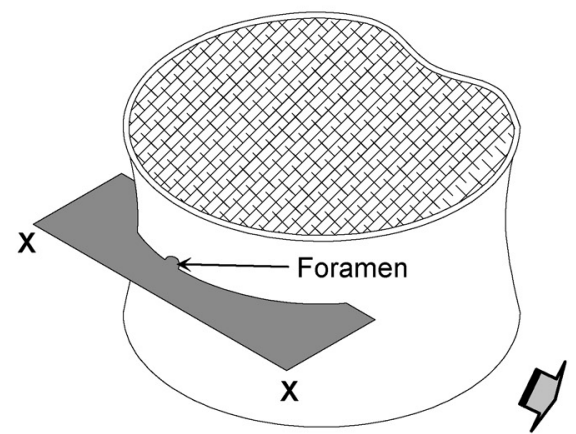

(a) Vertebral body

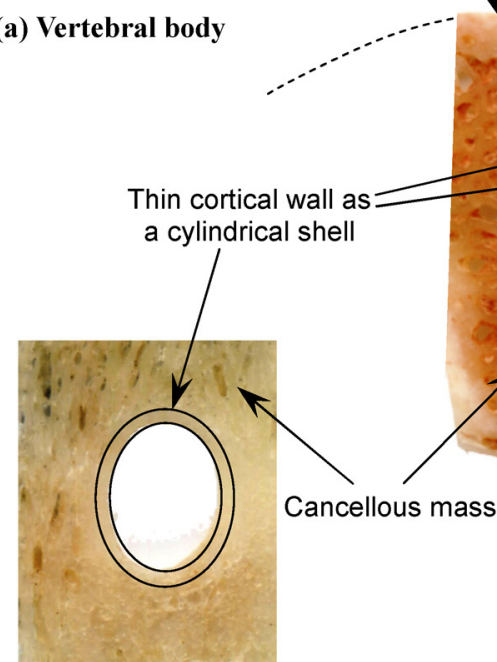

(c)

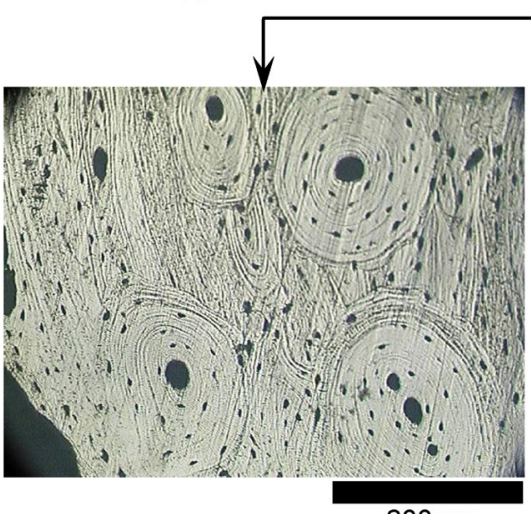

$200 \mu \mathrm{m}$

(d)
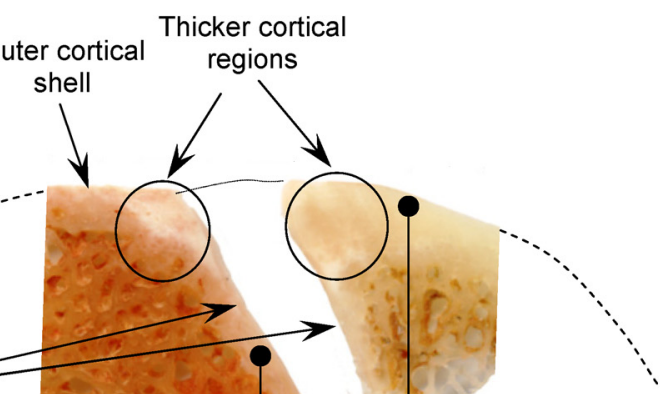

$2 \mathrm{~mm}$

(b) Section X-X

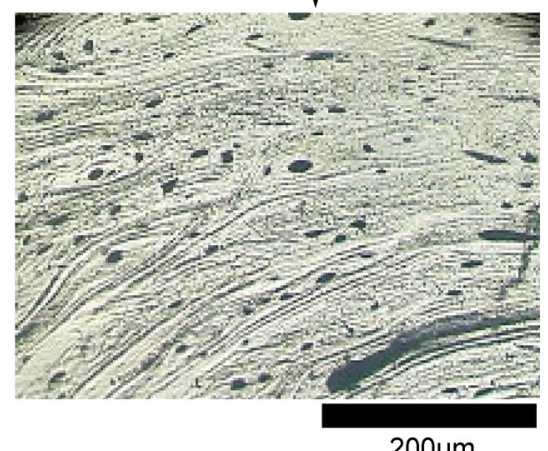

(e)

Fig. 7 (a) Vertebral body diagram with the foramen. (b) Section through the foramen center. Cortical mass is thicker at the upper foramen edge; cortical wall inside the cancellous mass indicates the existence of cylindrical shell covering the foramen from inside. (c) Sample remained with cancellous portion after slicing cortical bone specimen. A ring like cortical bone is still existed around the foramen representing the cortical cylindrical shell. (d) Microscopic image from the pointed location of the wall showing osteons pack aligned along the plane of the paper, indicating the circumferential alignment of osteons in the inner cylindrical body. (e) Microscopic image from the pointed location of the outer (surface) cortical shell showing linear alignment of the osteons. 


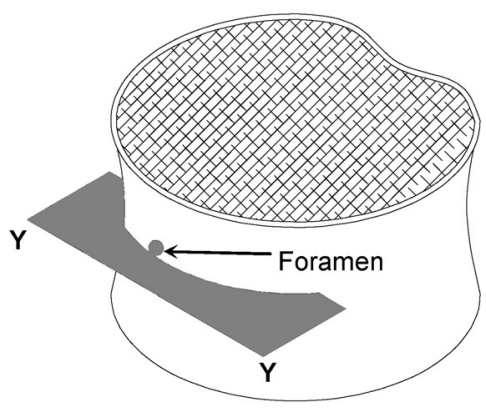

(a) Vertebral body

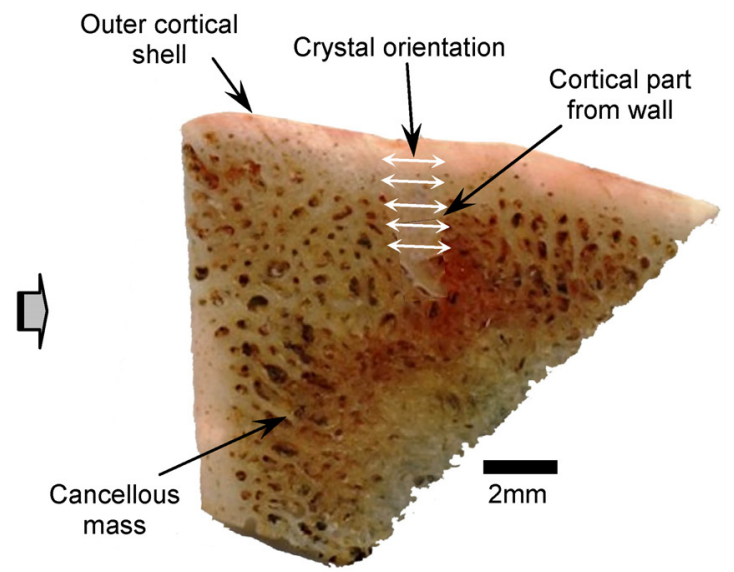

(b) Section Y-Y

Fig. 8 (a) Vertebral body indicating plane of section considered at the end of the foramen. (b) Crystal orientation on portion of cortical bone at the foramen end.

\section{Discussion}

X-ray diffraction method has been widely used to understand the structure, nature and function of bone tissue. The requirement of non-destructive and non-invasive method and the advantage of higher degree (Angstrom level) measurements have led to the extensive application of X-rays in the study of mineralized tissue. Tadano et al ${ }^{(8)-(10)}$ applied the diffraction profile of X-rays to measure stress in bone tissue. Fujisaki and Tadano et al ${ }^{(11)}$ considered the diffracted intensity profile of X-rays to measure bone tissue lattice strain.

HAp crystals are shown to be of good atomic order and well-ordered lattice, hence give a good diffraction pattern showing characteristic reflections. The 002-lattice plane of HAp crystals, which indicates $\mathrm{c}$-axis orientation, has sharper reflection compared to other planes and provides an estimate of the preferential direction (tendency of alignment) of crystallites c-axis. Some sort of radiation scattering occurs as a result of diffraction from different lattice planes and broadening of diffraction maxima because of crystals being inclined to the direction of incident beam, when considered three dimensionally ${ }^{(4)}$. So, the 002-plane in two dimensional representation of intensity distribution in our case (Fig. 3 (b)) may possess slight error in the orientation of crystals. However, this is expected to have negligible effect in the consideration of preferential orientation of c-axes.

For the bones of region like lumbar vertebra, where they are subjected to complex external loads, the alignment of collagen fibers (crystals) are expected to organize differently unlike the case of long bones (e.g. femur). Our curiosity to consider the specimen of lumbar region with the foramen was to investigate using X-rays for more precise and accurate results to know if the compositional parameters agree with the case of long bones. Previous works on stress concentration issues have shown effective use of tailored fiber orientations around the hole by reducing the stress concentration and increasing overall load carrying capacity of the structure. Optimization of fiber alignment around the hole for increased strength have shown that the optimum fibers approximately form concentric circles near the hole and gradually merge to far field fibers away from the hole ${ }^{(12)}$. The orientation of crystals revealed in this study reflects similar kind of structure around the naturally occurring hole, foramen (Fig. 4). The crystals arranged in curve like structure near the edge of the hole furnish a tailored structure altering the principal load direction, thereby reducing the local stress. Furthermore, considering the shape of the hole, elliptical has lower peak normal stress at the edge compared to circular shape. The foramina we observed are all more or less elliptical in shape. X-ray absorption method, generally 
considered as an accurate way to estimate the minerals ${ }^{(5)}$, revealed erratic distribution of the apparent mineral fraction in the specimens (Fig. 6). No specific tendency in the distribution of mineral fraction values indicated negligible role of mineral content in reducing the stress concentration around the foramen. Our findings in this case do not agree with the results previously reported ${ }^{(13)}$.

We, for the first time, explored some interesting facts from the examination of internal structure of the foramen. Though cortical bone is considered as a thin shell covering cancellous mass in vertebral bodies, a thicker cortical region was discovered around the upper part of the foramen at the edge near the external surface of the vertebral body compared to other regions (Fig. 7 (b)). Since cortical bone is considered as more damage resistant compared to trabecular bone, existence of such structure has protected the foramen additionally. Furthermore, the extension of cortical part inside the cancellous mass as a thin cylindrical shell (wall) (Fig. 7 (b) and (c)) having circumferentially organized crystals in a way to reinforce the hole strengthened the surface of the foramen causing the crack growth to deviate away from it. Osteons pack (Fig. 7 (d)) and horizontal alignment of crystals (Fig. 8 (b)) observed from the sections of cortical wall indicate the circumferential alignment around the foramen, whereas osteons near the external surface are linearly aligned (Fig. 7 (e)).

We have performed similar investigation with more than the presented specimens (i.e. 4 specimens for the crystal orientation and mineral distribution, and more than 5 specimens for the internal structure of the foramen, all from the three donors) and the results obtained in all were similar to those presented here with four samples. Hence, the outcomes obtained in this work could be generalized to the bovine population as a whole. Furthermore, primary focus of the current work is on the foramen of the regions where the loading is not unidirectional (spine sample in this case). Because of this, they are expected to behave differently from the case of long bones, where they are basically subjected with tensile or compressive loading only. So looking at the objective of the current work, we find it of less relevance comparing the cases of a quadruped and a human (where basically the principal loading direction differs) and relate the findings to the human response.

In the current work, we have explored the morphology of foramen region considering it as an independent hole. We have not considered the role of interaction between bone and blood vessels or nerves and flow of fluid within, which may have effect in the morphology of the foramen.

\section{Concluding Remarks}

The current work hence revealed novel facts regarding the cortical bone structure around the foramen existing in the lumbar vertebral body of bovine. We presented better and precise outcomes by the application of X-ray diffraction and X-ray absorption methods. The curvilinear shaped crystals at the surface near the edge as well as in the cylindrical wall of the foramen are regarded to reduce the stress concentration around the foramen edge resulting in an improved load bearing structure. The thicker cortical portion around the edge of the foramen near the surface has further resulted it a damage resistant structure. The mineral content, which controls the elastic properties of the bone, is not considered to effect the reduction of stress concentration around the foramen.

Except these, the method we introduced in this work to determine the crystal orientation not by observing the intensity pattern, but by quantifying the actual intensity values, gives more specific orientation result. This could ultimately be used to predict the anisotropic behavior of bone tissue, which is out of scope of the current work.

The methods described in this study and the results obtained may give new ways for further investigation of complex bone tissue. 


\section{Acknowledgement}

This work was supported by Grant-in-Aid for Scientific Research (B) (2), MEXT (No. 16300143), Japan.

\section{References}

(1) Sasaki, N. et al, Orientation of bone mineral and its role in the anisotropic mechanical properties of bone - transverse anisotropy, Journal of Biomechanics, Vol. 22, No. 2, pp. 157-164, 1989.

(2) Sasaki, N. and Sudoh, Y., X-ray pole figure analysis of apatite crystals and collagen molecules in bone, Calcified Tissue International, Vol. 60, No. 4, pp. 361-367, 1997.

(3) Currey, J.D., The effect of porosity and mineral content on the young's modulus of elasticity of compact bone, Journal of Biomechanics, Vol. 21, No. 2, pp. 131-139, 1998.

(4) Eanes, E.D., X-ray diffraction of vertebrate hard tissue, In: Zipkin, I. (Ed.), Biological Mineralization, John Willey \& Sons, New York, pp. 227-256, 1973.

(5) Vose, G.P., Determination of organic-inorganic ratio in osseous tissue by X-ray absorption., Analytical Chemistry, Vol. 30, No. 11, pp. 1819-1821, 1958.

(6) Koch, B. et al, Absorption, In: Lonsdale, K. (Ed.), International Tables for X-ray Crystallography, Vol. 3, The Kynoch Press, UK, pp. 157-200, 1962.

(7) Cullity, B.D. and Stock, S.R., Elements of X-ray Diffraction, Prentice-Hall, USA, 2001.

(8) Tadano, S. and Okoshi, T., Residual stress in bone structure and tissue of rabbit's tibiofibula, Bio-Medical Materials and Engineering, Vol. 16, No. 1, pp. 11-21, 2006.

(9) Tadano, S. and Todoh, M., Anisotropic residual stress measurements in compact bone using polychromatic X-ray diffraction, In: Pedersen, P., Bendsøe, M.P. (Eds.), IUTAM Symposium on Synthesis in Bio Solid Mechanics, Kluwer Academic Publications, Pordr Netherland, pp. 139-159, 1999.

(10) Todoh, M. et al, Polychromatic X-ray measurements of anisotropic residual stress in bovine femoral bone, JSME International Journal - Series C, Vol. 43-44, pp. 795-801, 2000.

(11) Fujisaki, K. and Tadano, S. et al, A method on strain measurement of HAP in cortical bone from diffusive profile of X-ray diffraction, Journal of Biomechanics, Vol. 39, No. 3, pp. 579-586, 2006.

(12) Huang J. and Haftka R.T., Optimization of fiber orientations near a hole for increased load-carrying capacity of composite laminates, Structural and Multidisciplinary Optimization, Vol. 30, No. 5, pp. 335-341, 2005.

(13) Götzen, N. et al, Understanding stress concentration about nutrient foramen, Journal of Biomechanics, Vol. 36, No. 10, pp. 1511-1521, 2003. 